\title{
Adenovirus en Niños Menores de Cinco Años. Patrones de Circulación, Características Clínicas y Epidemiológicas en Colombia, 1997-2003
}

\author{
Adenovirus in children under five years of age. Circulation patterns \\ and clinical and epidemiological characteristics \\ in Colombia, 1997-2003 \\ Diana H. Herrera-Rodríguez ${ }^{1}$, Fernando de la $\mathrm{Hoz}^{2}$, Cristina Mariño ${ }^{3}$, Eliana \\ Ramirez ${ }^{3}$, Juan D. López ${ }^{4}$ y Consuelo Vélez ${ }^{4}$ \\ 1 Grupo de Virologia; Instituto Nacional de Salud. Bogotá, Colombia. diana_herrerar@yahoo.es \\ 2 Departamento de Salud Pública. Facultad de Medicina Universidad Nacional de Colombia. \\ fpdelahozr@unal.edu.co. \\ 3. Departamento de Pediatria. Hospital Militar Central, Bogotá, Colombia. \\ 4 Secretaria de Salud. Manizales, Colombia
}

Recibido 8 Agosto 2006/Enviado para Modificación 31 Marzo 2007/Aceptado 24 Agosto 2007

\section{RESUMEN}

Objetivo Desde 1997 el Instituto Nacional de Salud vigila la ocurrencia de algunos virus respiratorios con el fin de identificar sus patrones de circulación y el papel que tienen en la ocurrencia de enfermedad respiratoria moderada o severa, en niños menores de 5 años.

Métodos La vigilancia está basada en hospitales centinela de Bogotá y Manizales en los que todas las semanas se recolectan 4 a 8 muestras nasofaríngeas, de niños menores de cinco años que consultan por enfermedad respiratoria alta o baja. Las muestras son procesadas por inmunofluorescencia indirecta.

Resultados De Enero de 1997 a Diciembre de 2003 se examinaron 1743 pacientes y se encontraron virus respiratorios en $35 \%$ de las muestras procesadas. El adenovirus se encontró en 47 casos (2,6 \%, IC95 \% 1,4-3,5\%) y fue detectado durante todos los meses, excepto enero, con picos en marzo, julio, octubre y diciembre. La mayoría de los pacientes requirieron hospitalización (50 a $87 \%$ ) y soporte con oxigeno y la razón muerte/caso fue de 7\%. Los serotipos de adenovirus encontrados fueron B7, 2, C1 y B3.

Conclusiones Los datos del presente estudio datos sugieren que en nuestro medio los adenovirus no son los agentes mas frecuentes de enfermedad respiratoria pero, cuando están presentes, producen enfermedad severa.

Palabras Clave: Adenovirus, epidemiología, Colombia (fuente: DeCS, BIREME). 


\section{ABSTRACT}

Objective The Colombian National Institute of Health has been studying the epidemiology of several respiratory viruses since 1997 to identify their circulation patterns and the role they play as pathogenic agents of acute and/or moderate respiratory infection (RI) in children aged less than 5 .

Methods The study used sentinel surveillance methods in sentinel hospitals in Bogotá and Manizales. Four to eight nasopharyngeal samples were collected every week in the sentinel hospitals from children aged less than five consulting for low or high RI. Nasopharyngeal samples were processed by indirect immunofluorescence. Results 1743 children were examined from January 1997 to December 2003; $35 \%$ were found to be positive for respiratory viruses. Respiratory syncytial virus was found more frequently while adenovirus was found in 47 patients $(2,6 \%$ of the sample; 1,4-2,5\% $95 \% \mathrm{Cl}$ ). Adenovirus was detected throughout the year (excepting January) but peaks occurred in March, July, October and December. Most patients were hospitalised (50-87\%) and were in need of oxygen support. Fatality:case ratio was $7 \%$; B7, 2, C1, and B3 serotypes were identified.

Conclusion The data suggested that respiratory infection due to adenovirus was a low frequency event amongst the children seeking medical care at the sentinel hospitals. However, when respiratory disease was caused by adenovirus, it was severe.

Key Words: Adenoviridae, epidemiology, children, Colombia (source: MeSH, NLM).

$\mathrm{L}$ as infecciones respiratorias agudas (IRA) del tracto respiratorio inferior son una de las principales causas de mortalidad de niños en el mundo, particularmente en países en desarrollo, causando aproximadamente un tercio de todas las muertes estimadas en niños menores de 5 años. El promedio de episodios de infección respiratoria al año es de 12 en niños menores de 5 años y aproximadamente 3 en adultos (1).

Entre los numerosos agentes etiológicos descritos, ya sea en países en desarrollo o en países industrializados los virus se reconocen como los agentes predominantes en las IRA y entre ellos los adenovirus afectan especialmente a niños y a personal militar $(2,3)$.

Aproximadamente $65 \%$ de los aislamientos de adenovirus son en niños menores de 4 años causando el $10 \%$ de las infecciones respiratorias que requieren hospitalización en este grupo de edad.

Los adenovirus pueden causar faringitis, conjuntivitis, laringotraqueobron quitis y bronquiolitis, pero las neumonías agudas son, sin lugar a dudas, las 
manifestaciones clínicas más graves, sobre todo en los niños pequeños en los que pueden ser fatales. Los tipos más frecuentemente asociados a estos cuadros son Ad3, Ad4, Ad7, Ad21, así como Ad2 y Ad5. Se han descrito las secuelas de daño pulmonar residual importante como bronquiectasias y bronquiolitis (1,4$6)$.

En Colombia hay poca información sobre los patrones de comportamiento de las infecciones respiratorias y su relación con la circulación de adenovirus, como también las complicaciones clínicas que este virus produce en niños menores de 5 años. Debido a ello el Instituto Nacional de Salud (INS) empezó la vigilancia de la frecuencia de algunos virus respiratorios en la población desde 1997 con el objetivo de identificar el patrón de circulación de influenza, parainfluenza 1, 2 y 3, adenovirus y el virus sincitial respiratorio (VSR) $(7,8)$.

El presente artículo analiza los resultados de la vigilancia para el adenovirus entre 1997 y 2003 en los centros centinelas de las ciudades de Manizales, Bogotá y de otras ciudades del país que participan en la vigilancia cuando ocurren brotes de enfermedad respiratoria.

\section{MATERIALES Y MÉTODOS}

Este es un estudio descriptivo basado en casos de infección respiratoria que consultaron a centros de vigilancia centinela para virus respiratorios en el país.

La muestra estaba compuesta de niños menores de cinco años que consultaron a los centros centinelas de Bogotá y Manizales los días Martes entre Marzo de 1997 y diciembre de 2003, con sintomatología compatible con infección respiratoria y a quienes se tomó muestra para identificación viral. Se incluyeron pacientes con menos de cuatro días de evolución del cuadro clínico y con al menos dos de los siguientes síntomas: fiebre, tos, dolor de garganta o rinorrea. Cada martes, en los centros de vigilancia, se seleccionaban de 3 a cinco pacientes entre los primeros que consultaban a los servicios de urgencia o de consulta externa y que cumplían los criterios de selección. Durante el período de vigilancia también se recibieron muestras de otras ciudades del país que no realizan una vigilancia permanente pero que envían muestras cuando detectan aumentos en el número de casos de infección respiratoria.

Las muestras se tomaron en medio de transporte para virus respiratorios (Voricult ${ }^{\circledR}$ (MW 950/974/975 Medical Wire Equipment Co. Ltd.) de la pared posterior de la faringe, idealmente dirigida hacia la nasofaringe y enviadas al 
laboratorio dentro de las primeras 48 horas posteriores a la toma de la muestra. En el laboratorio de Virologia del INS se procesaron por inmunofluorescencia indirecta (Panel Respiratorio Viral. Catalogo 3105, Chemicon) (9), se realizó intento de aislamiento viral en células H 292 y se confirmo el aislamiento por inmunofluorescencia. Los aislamientos positivos fueron enviados al CDC de Atlanta para determinar el serotipo viral.

Las fuentes de información que se consultaron para extraer las variables clínicas y epidemiológicas de los pacientes incluían a las fichas epidemiológicas utilizadas por los centros centinelas, las historias clínicas y los registros de laboratorio de virología del Instituto Nacional de Salud. Las variables recolectadas fueron: sexo, procedencia, síntomas (fiebre, tos, rinorrea, odinofagia, cefalea, mialgias, dificultad respiratoria, vomito, otalgia), diagnostico clínico, manejo (ambulatorio, hospitalario), hospitalario (UCI, intubación), egreso (mejoría defunción dependencia de oxigeno), tratamiento antimicrobiano, diagnostico virológico, edad y días de estancia hospitalaria y el nivel socioeconómico.

Los datos de los pacientes y los resultados del laboratorio se digitaron en una base de datos del programa estadístico Epi.info 6.04 (10). Se describió la frecuencia del adenovirus con respecto al total de los pacientes observados y para cada uno de los cuadros clínicos descritos en las historia clínicas. Para la descripción de las variables se utilizaron medidas de tendencia central como el promedio, de posición como la mediana, o porcentajes de acuerdo con la escala de medición de las variables de interés. Cuando fue necesario se calcularon intervalos de confianza y las diferencias de proporciones o de promedios fueron evaluadas con pruebas de significancia estadística como Chi cuadrado, test exacto de Fisher o análisis de varianza según el caso. Para evaluar la magnitud de las asociaciones y su significancia estadística se usaron las razones de Ventaja. Para todos los intervalos de confianza o pruebas estadísticas el nivel de confianza fue del $95 \%$.

\section{RESULTADOS}

De Enero de 1997 a Diciembre de 2003 se procesaron 1743 muestras de pacientes con infección respiratoria aguda que tomaron parte en la vigilancia centinela de influenza y otros virus respiratorios en Colombia. El adenovirus se identificó en 47 casos (2.6 \%) (IC $95 \%$ 1,4-3,5 \%), mientras que otros virus respiratorios como virus de influenza A y B, Virus sincitial respiratorio y parainfluenza 1, 2 y 3 fueron identificados en un $35 \%$ de las muestras procesadas. 
La mayor parte de los pacientes a los que se les identificó adenovirus provenían de la ciudad de Bogotá 37 (79 \%), 4 (8,4 \%) de Manizales y el resto de Medellín 1 (2,1\%), Soacha 1 (2,1\%), y Tolemaida 1 (2,1\%). Tres pacientes no tenían dato de procedencia (6,3\%). El 71,4 \% eran del sexo masculino y la mediana de edad de los casos fue de siete meses. Treinta pacientes (68 \%) eran menores de un año y dentro de este grupo se encontró la mayor proporción de aislamientos (6,4\%), como se indica en la Tabla 1.

Tabla 1. Muestras de Adenovirus por grupos de edad Colombia, 1997-2003.

\begin{tabular}{lccc}
\hline $\begin{array}{c}\text { Grupos edad } \\
\text { meses }\end{array}$ & $\begin{array}{c}\text { Muestras } \\
\text { procesadas }\end{array}$ & $\begin{array}{c}\text { Muestras } \\
\text { positivas }\end{array}$ & $\begin{array}{c}\% \\
\text { positivas }\end{array}$ \\
\hline$<6$ a & 713 & 21 & 2,9 \\
7 a 11 & 255 & 9 & 3,5 \\
12 a 24 & 436 & 7 & 1,6 \\
25 a 60 & 225 & 8 & 3,5 \\
Sin dato & 112 & 2 & 1,8 \\
Total & 1743 & 47 & 2,6 \\
\hline
\end{tabular}

Los síntomas más frecuentes en los pacientes con adenovirus fueron: Tos $91 \%$ (40/44), fiebre $60 \%$ (26/43), dificultad respiratoria $56 \%$ (23/41), y rinorrea 49 \% (19/43). Los diagnósticos clínicos más frecuentes fueron: neumonía en un $28 \%$ (13/47), bronconeumonía en un $23 \%$ (11/47), bronquiolitis en un $19 \%$ (9/47), síndrome coqueluchoide en un 17 \% (8/47) e IRA alta en 13 \% (6/47).

Durante los seis años de vigilancia se observó un incremento en la circulación de Adenovirus en los meses de Marzo, Julio, Octubre y Diciembre mientras que en Enero no se detectaron casos. La mayor circulación se presento en el año 1998 (Figuras 1 y 2).

Figura 1. Numero de adenovirus identificados por mes en Colombia. 1997-2003.

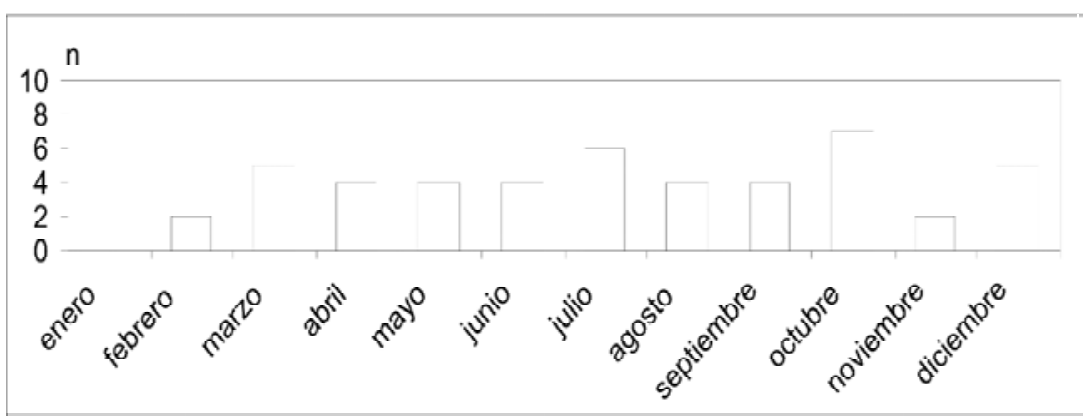

De los 47 pacientes a los que se les identifico adenovirus se tuvieron datos sobre el manejo clínico en 23 (48 \%). El 87 \% (20/23) fueron hospitalizados y 
$13 \%(3 / 23)$ recibieron tratamiento ambulatorio. Después de ser atendidos en el hospital, 21/23 (91 \%) fueron tratados con antibióticos. El uso de antibióticos era mas frecuente en pacientes con cianosis (6/8) que en pacientes sin cianosis (16/36).

Figura 2 . Numero de adenovirus identificados por año en Colombia. 1997 - 2003

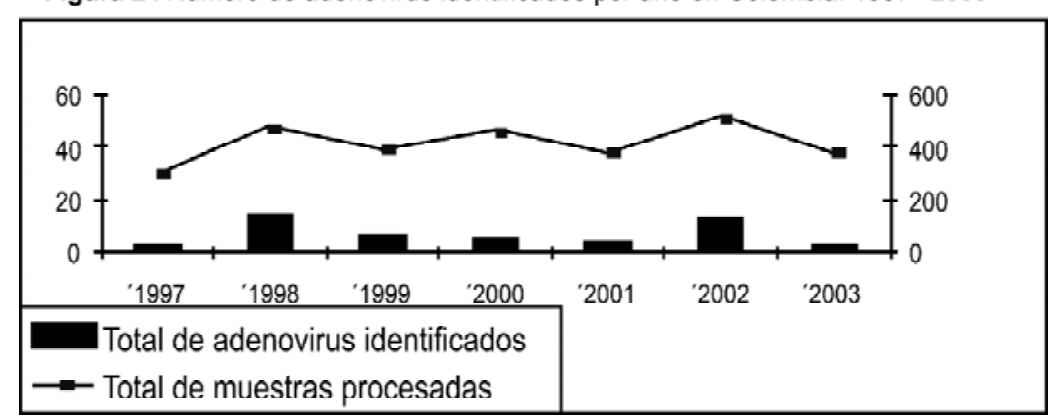

En 52 \% (12/23) de los pacientes hospitalizados se obtuvo información sobre los días de estancia que estuvieron entre 7 y 32 días con un promedio de 13 días. El 83 \% (10/12) ingresaron en la Unidad de Cuidados intensivos (UCI) con un promedio de estancia de 10 días, 11 (91\%) pacientes requirieron soporte de oxigeno y 4 tuvieron que ser intubados (33 \%). La hospitalización en UCI no estaba relacionada con la edad, pero si con la presencia de dificultad respiratoria y con la toma de antibióticos antes del ingreso al hospital. La probabilidad de ser internado en UCI fue 10 veces mayor en los que tenían dificultad respiratoria (OR $=10.9$ CI95 \% 1,1-259) y 7 veces mayor para los que estaban tomando antibióticos antes de ser atendidos (OR $=7,2$ CI95 \% $0,6-194, p=0,08)$.

Los hallazgos radiológicos más frecuentes fueron infiltrados, en 91 \% (11/ 12) de los pacientes, atrapamiento de aire en $50 \%$ (6/12), consolidaciones, especialmente de los lóbulos superiories, en $42 \%$ (5/12), y atelectasias en 8 $\%(2 / 12)$ de los pacientes.

Del $65 \%(13 / 20)$ de los pacientes había informe de su estado al egreso habiendo fallecido uno de ellos (7,7 \%). Este paciente tenía un año de edad e ingresó con un diagnostico de bronquiolitis y croup severo. Durante la hospitalización presentó complicaciones como neumonía derecha, sepsis y falla renal. Estuvo 14 días hospitalizado y requirió intubación e ingreso a UCI. 
En el $27 \%$ (8/30) de los casos se logró aislar y caracterizar el serotipo de adenovirus. El 50 \% (4/8) fueron identificados como B7, $25 \%(2 / 8)$ se identificaron como adenovirus 2, 12,5 \% (1/8) como C1 y 12,5 \% (1/8) como B3. Uno de los pacientes con B 7 fue hospitalizado y estuvo 9 días interno, egresando sin presentar complicaciones y sin ingresar a UCI.

\section{DISCUSIÓN}

En Latinoamérica existe un vacío importante en el conocimiento de la epidemiología del adenovirus, especialmente en la región Andina y Centroamérica. En la búsqueda bibliográfica que se realizo alrededor de este estudio solo se encontraron 9 estudios publicados en América Latina con algún tipo de análisis sobre el comportamiento o las características del adenovirus. Estos estudios eran de Brasil, Argentina y Chile y habían sido conducidos en hospitales por periodos de tiempo más cortos que el presente estudio y, con tamaños de muestra más pequeños (5-6, 11-16).

La principal fortaleza de este estudio es que la vigilancia de los virus respiratorios se ha llevado a cabo en un amplio número de pacientes y por un periodo de tiempo relativamente largo, de manera que aporta información importante para llenar parcialmente un vacío de información sobre la etiología de la infección respiratoria grave y moderada en el país y en la región. En primer lugar, los datos del presente estudio sugieren que el adenovirus es un agente de baja frecuencia entre los niños que consultan por IRA severa o moderada a hospitales de tercer nivel lo cual coincide con algunos estudios realizados sobre este tema (11-16). La distribución del virus por síndrome clínico es similar a lo reportado en la literatura, siendo más frecuente en neumonía, bronquiolitis y síndrome coqueluchoide.

En segundo lugar, el estudio también sugiere que no hay un patrón estacional claro de circulación de este virus ya que se encontró durante todo el año, a excepción de enero, época en que generalmente los casos de IRA disminuyen en el todo el país. Otros hallazgos de Latinoamérica muestran algunas diferencias con el patrón encontrado en Colombia. En dos estudios de Brasil, uno en Salvador (BA) y otro en Portoalegre, encontraron que el adenovirus predominaba en el segundo semestre del año y que muy rara vez se encontraba en el primer semestre. Sin embargo, esta diferencia puede deberse a que en los estudios brasileños se tomaban mas muestras durante los meses del invierno del hemisferio sur (Mayo a Septiembre), mientras que en Colombia se tomaron muestras todos los meses durante los años del estudio. Por el contrario, en 
estudios de Taiwán y Corea se encontró también que el adenovirus circulaba durante todo el año $(12,13,17,18)$. De todas maneras no puede descartarse completamente un comportamiento estacional entre nosotros, dado el numero bajo de aislamientos identificados.

Otro hallazgo importante es que, a pesar de su baja frecuencia relativa, los presentes hallazgos confirman que la enfermedad producida por este agente tiende a ser severa. Los hallazgos que mas apoyan esta conclusión son: El 50 y el $90 \%$ de los pacientes tuvieron que ser hospitalizados, la estancia hospitalaria estuvo por encima de los 7 días en la mayoría de ellos, la mayoría de los pacientes necesito soporte con oxigeno y un porcentaje importante de pacientes ingresó a la unidad de cuidados intensivos con un promedio de estancia prolongado (10 días o más). Esto coincide con otros estudios donde se ha encontrado al adenovirus asociado con enfermedades respiratorias agudas fatales y casi fatales, así como con la presencia de bronquiolitis obliterante $(5,6,19)$.

El presente estudio también permite aproximarse a la identificación de los serotipos de adenovirus causantes de enfermedad que requiere hospitalización. Las resultados de la serotipificación sugieren que el adenovirus tipo B7 es el agente más frecuente de cuadros respiratorios que ameritan consulta a hospitales, lo que coincide con datos de estudios alrededor del mundo que muestran como la mayoría de las infecciones respiratorias severas causadas por adenovirus en niños menores de un año, son debidas al adenovirus tipo B 7 $(14,16,20-22)$.

Los resultados de este estudio podrían servir a las instituciones participantes en el sistema de seguridad social para implementar en servicio de diagnóstico de virus respiratorios en los hospitales ya que muchos pacientes son inadecuadamente manejados por desconocimiento de la etiología del cuadro respiratorio. El diagnóstico adecuado de la etiología de la enfermedad respiratoria permitiría la disminución del uso de antibióticos, probablemente la disminución de la resistencia bacteriana y mejorar el conocimiento sobre la epidemiología de la enfermedad respiratoria severa en Colombia.

Como todo estudio basado en datos de vigilancia, este presenta algunas limitaciones. Una de ellas es que las muestras procedieron en su mayoría de dos ciudades, Bogotá y Manizales, lo que dificulta extrapolar algunos resultados al resto del país, especialmente en lo que tiene que ver con el patrón temporal de circulación de este virus. Sin embargo, hallazgos como la severidad de la 
enfermedad asociada con adenovirus, los serotipos más frecuentes y el peso de estos virus como agente causal de enfermedad moderada o severa en menores de cinco años pueden ser generalizables.

Otro problema potencial fue la alta frecuencia de muestras no aptas para intento de aislamiento viral (36 \%) y por lo tanto no fue posible serotipificar a todos los adenovirus identificados por inmunofluorescencia. Esto impide determinar con precisión todos los posibles efectos clínicos de la infección por adenovirus e identificar los serotipos que se asocian con enfermedad más severa. Adicionalmente, en varios pacientes no se tuvo información para variables de interés clínico y epidemiológico debido al diligenciamiento incompleto de las historias clínicas

\section{REFERENCIAS}

1. Horwitz M.. Adenoviral diseases. In: Virology, Fields BN, MelnickJL(Eds.), New York: Rave Press; 1985. pp 477-495

2. CDC. Two fatal cases of adenovirus related illness in previously healthy young adults. MMWR2001; 50 (26):553-555

3. Mills McN, Benton R, Monteith S, Tuchscherer M, Gaydos J. Epidemic spread of adenovirus type 4 associated acute respiratory disease between US Army installations. Emerging Infectious Diseases 2000; 6(4):415-419.

4. Kajon A. Adenoviridae. En: Virología Médica, Caballal G, Oubiña JR (Eds.). Buenos Aires: Editorial el Ateneo; 1991. pp 273-80

5. Arce J, Moncada R, Mardones R, Velozo L, Parra G. Secuelas post-infección por adenovirus en niños: Evaluacion con tomografía computada. Revista Chilena de Radiología 2002; 8 (4): 154-163.

6. Fisher G, Teper A, Colom A. Acute viral bronchiolitis and its sequelae in developing countries. Pediatric Respiratory Reviews 2002; 3: 298-302

7. Herrera D, De la Hoz F, Mariño C, López JD, Vélez C, Arboleda LM. Vigilancia de influenza y virus respiratorios en Colombia enero de 2000 a 31 de julio de 2001, Informe quincenal Epidemiológico Nacional 2001; 6: (17): 267-72.

8. Herrera D, De la Hoz F, Mariño C, López J, Vélez C, Arboleda L. Vigilancia de influenza y virus respiratorios en Colombia, 2002. Informe quincenal Epidemiológico Nacional; 8: (19): 301-5.

9. McDonald JC, Quennec P. Utility of a respiratory virus panel containing a monoclonal antibody pool for screening of respiratory specimens in nonpeak respiratory syncitial virus season. JClin Microbiol 1993;31(10):2809-2811. 
10. Dean AG, Dean JA, Coulombier D. Epi Info, version 6: a word prosessing, database, and statistics program for public health on IBM compatible microcomputers. Atlanta, Georgia, U.S.A: Centers for Disease Control and Prevention; 1995.

11. Savy V, Baumeister E, Boir F, Shiroma M, Campos A. Evaluación etiológica y clínica de infecciones respiratorias agudas bajas en una población infantil. Medicina (Buenos Aires) 1996; 56(3): 213-217.

12. Straliotto S, Siquiera M, Muller R, Fischer G, Cunha M, Nestor S. Viral etiology of acute respiratory infections among children in Porto Alegre, RS, Brazil. Revista da Sociedade Brasileira de Medicina Tropical 2002; 35 (4):283-291.

13. Moura E, Borges L, Souza L, Ribeiro D, Siqueira My Ramos E. Estudo de infeccoes respiratorias agudas virais em criamcas atendidas en um centro pediatrico em Salvador (BA). Jornal Brasileiro de Patología e Medicina Laboratorial 2003; 39 (4):275-282.

14. Celadilla M, Winocur D, Cohen et al. Virus Sincitial Respiratorio en menores de cinco años hospitalizados. Infectología y Microbiología Clínica 1992; 4: 31-5.

15. Pumariega T, Savón C, Muné M, Cancio R, González G, Valdivia A, et al Isolation and identification of adenovirus in hospitalised children under five years, with acute respiratory disease, in Havana, Cuba. Mem Inst Oswaldo Cruz 2000; 95 (6):859861

16. Murtagh P, Cerqueiro C, Halac A, Avila M, Kajon A. Adenovirus type 7 h respiratory infeccions: a report 29 cases of acute lower respiratory disease. Acta Paediatrica 1993; 86(6-7): 557-561.

17. Kim M, Lee H, Lee G Epidemiology of acute viral respiratory tract infections in Korean children. Journal of Infection 2000; 41:152-58.

18. Lin T, Huang Y, Ning H, Tsao K. Surveillance of respiratory virus infections among pediatric outpatients in Northern Taiwan. Journal of Clinical Virology 2004; 30 : 81-5.

19. Tan W, Xiang X, Qiu D, Pin Ng T, Lam S, Hegele R. Epidemiology of respiratory viruses in Patients hospitalised with near fatal asthma, acute exacerbations of asthma, or chronic obstructive pulmonary disease. The American Journal of Medicine2003; 115: 272-277.

20. Straube RC, Thompson MA, Van Dyke RB, Wadell G, Connor JD, Wingard D, Spetor SA. Adenovirus type 7b in a children's hospital. Journal of Infectious Diseases 1983; 147(5): 814-819.

21. Day A, McGregor D, Henderson S, Teele D. Fatal adenoviral disease in siblings. Pediatric Infectious Disease Journal 1998; 17 (1): 83 - 85.

22. Avila M, Carballal G, Rovalentti H. Viral etiology in Acute Lower Respiratory Infection in children from a close community. American Rev. Respir Dis 1989; 140: 634-7. 